\title{
SIMULATION OF ELECTRIC ARC WITH REFRACTORY CATHODE AND EVAPORATING ANODE
}

\author{
I.V. KRIKENT ${ }^{1}$, I.V. KRIVTSUN ${ }^{2}$ and V.F. DEMCHENKO ${ }^{2}$ \\ ${ }^{1}$ Dneprodzerzhinsk State Technical University \\ 2 Dneprostroevskaya Str., 51918, Dneprodzerzhinsk, Ukraine. E-mail: science@dstu.dp.ua \\ ${ }^{2}$ E.O. Paton Electric Welding Institute, NASU \\ 11 Bozhenko Str., 03680, Kiev, Ukraine. E-mail: office@paton.kiev.ua
}

\begin{abstract}
Equation of convective diffusion of ionized metal vapour in arc plasma, allowing for the difference in coefficients of diffusion of atoms, single- and double-charged metal ions, presence of thermodiffusion flows of metal particles, as well as ion drift in the electric field, was proposed to more precisely define the earlier developed complex model of the processes of energy, mass and charge transfer in the column and anode region of electric arc with refractory cathode and evaporating anode, running in inert gas. Based on the thus precised complex mathematical model, numerical analysis of the influence of diffusion-induced evaporation of anode material ( $\mathrm{Fe}$ ) on heat, gas-dynamic and electromagnetic characteristics of multicomponent plasma of the column and anode region of stationary electric arc with refractory cathode (W) at its running in inert gas $(\mathrm{Ar})$ was performed. An essential influence of metal surface temperature distribution in the region of anode binding of the arc on distribution of temperature and electric current density in near-anode plasma, as well as on distributed and integral characteristics of its thermal impact on evaporating anode surface, is shown. 18 Ref., 12 Figures.
\end{abstract}

$\boldsymbol{K} \boldsymbol{e} \boldsymbol{y} \boldsymbol{w} \boldsymbol{O} \boldsymbol{r} \boldsymbol{d} \boldsymbol{s}:$ electric arc, refractory cathode, evaporating anode, arc column, anode region, multicomponent plasma, metal vapour, diffusion, mathematical simulation

Electric arc plasma in inert-gas nonconsumableelectrode welding, as a rule, is multicomponent, as alongside shielding gas particles, it contains atoms and ions of metal vapour coming to the arc gap due to evaporation of anode metal from weld pool surface. Presence of an even small amount of metal component in inert gas arc plasma has an essential influence on its ionization composition, thermodynamic, transport and optical properties. It leads to a significant difference of heat, electromagnetic and gas-dynamic characteristics of plasma in near-anode zone of arc column in nonconsumable-electrode welding from respective characteristics of arc discharge with refractory cathode and nonevaporating, for instance, water-cooled anode. Characteristics of welding arc anode region, determining the conditions of thermal and electromagnetic interaction of the arc with metal being welded and, consequently, nature of its penetration, are also different [1].

In the first publications, devoted to mathematical simulation of the processes of heat, mass and charge transfer in refractory cathode arcs [2-10], arc plasma was assumed to be single-com-

๑ I.V. KRIKENT, I.V. KRIVTSUN and V.F. DEMCHENKO, 2014 ponent, i.e. containing atoms and ions of just the shielding gas. Such idealization did not incorporate any conditions of running of real welding arcs, and required further improvement of mathematical models of the arc, in order to allow for a number of additional physical factors, related to multicomponent nature of arc plasma. Publications devoted to allowing for evaporation of anode material in simulation of nonconsumableelectrode welding arc, appeared in the world scientific literature comparatively recently [11-13]. When describing diffusion of ionized metal vapour in arc plasma, however, these works do not differentiate between vapour atoms and ions, having diffusion coefficients, differing significantly by their magnitude [1]. The integrated mathematical model of nonconsumable-electrode welding arc proposed in [14] is an attempt to allow for the difference in the above coefficients. However, when writing the equation of convective diffusion of evaporated anode metal in arc column plasma, thermodiffusion of atoms and ions of metal vapour, as well as metal drift in the electric field, were not allowed for. Therefore, the objective of this work is improvement of the model of convective diffusion of metal vapour in arc plasma and applying the precised integrated mathematical model [14] for numerical analysis of characteristics of multicomponent plasma of the column and anode region of sta- 
tionary electric arc with refractory (W) cathode and evaporating $(\mathrm{Fe})$ anode at its running in inert gas ( $\mathrm{Ar})$.

Equation of metal vapour transfer in arc plasma. A specific feature of metal vapour diffusion in arc plasma is that metal atoms evaporated from molten anode surface, may ionize, forming single- and double-charged ions, whose coefficients of diffusion differ significantly from the respective coefficients for neutral particles. More over, processes of ionization and recombination of particles in arc plasma column proceed much faster than those of transfer of substance and thermal energy $[15,16]$. Therefore, we will assume that concentrations of all the particles of multicomponent plasma of the considered arc column (electrons, atoms and single-charged ions of argon, atoms, single- and double-charged ions of iron) differ only slightly from equilibrium values determined by the principle of detailed equilibrium. We will also assume that arc column plasma is in the state of local thermodynamic equilibrium at electron temperature equal to that of heavy particles (single-temperature model).

In the general case the equation of diffusion for atoms, single- and double-charged metal ions in inert gar plasma can be expressed as follows:

$$
\begin{gathered}
\frac{\partial n_{m 0}}{\partial t}=-\operatorname{div}\left(n_{m 0} \vec{w}_{m 0}\right)-\dot{n}_{m 0} ; \\
\frac{\partial n_{m 1}}{\partial t}=-\operatorname{div}\left(n_{m 1} \vec{w}_{m 1}\right)-\dot{n}_{m 1}+\dot{n}_{m 0} ; \\
\frac{\partial n_{m 2}}{\partial t}=-\operatorname{div}\left(n_{m 2} \vec{w}_{m 2}\right)+\dot{n}_{m 1},
\end{gathered}
$$

where $n_{m 0}, n_{m 1}, n_{m 2}$ are the concentrations of atoms, single- and double-charged metal ions; $\vec{w}_{m 0}, \vec{w}_{m 1}, \vec{w}_{m 2}$ are the rates of their directed motion; $\dot{n}_{m Z}(Z=0,1)$ are the reaction rates of first and second ionization, respectively.

Let us represent the rate $\vec{w}_{m 0}$ of metal atom motion in the form of a sum of mean velocity $\vec{w}_{C}$ of plasma particle motion and diffusion rate of neutral metal particles $\vec{w}_{D 0}$ :

$$
\vec{w}_{m 0}=\vec{w}_{C}+\vec{w}_{D 0} .
$$

If atomic masses of all the plasma components are the same, then average velocity of particle motion coincides with average mass (gas dynamic) velocity of plasma motion $\vec{W}$. Otherwise, $\vec{w}_{C}$ value can be determined from the following balance relationship:

$$
\rho \vec{W}=\rho \vec{w}_{C}+M_{m} \vec{Y}_{m 0}+\bar{M}_{m 0} \vec{Y}_{\overline{m 0}},
$$

where $\rho$ is the plasma velocity; $M_{m}$ is the mass of metal atoms; $\vec{Y}_{m 0}$ is the density of diffusion flow of metal atoms; $\vec{Y}_{\overline{m 0}}, \bar{M}_{m 0}$ is the density of diffusion flow and average statistical mass of particles, substituting metal atoms.

As diffusion processes do not have any essential influence on pressure distribution in arc plasma, we can assume that it is determined, mainly, by gas-dynamic factors. For the considered here stationary free-running arc the pressure in its column differs only slightly from the atmospheric one [17]. Therefore, metal vapour diffusion in such an arc can with a high degree of accuracy be considered as a process running at constant (atmospheric) pressure. In this case, the result of diffusion is plasma-forming particles exchanging their places. It follows from here that specific diffusion flows $\vec{Y}_{m 0}$ and $\bar{Y} \overline{m 0}$ compensate each other, i.e. condition $\vec{Y}_{m 0}=-\vec{Y}_{m 0}$ is fulfilled. Then, equation (5) yields the following expression for determination of mean velocity of particle motion:

$$
\vec{w}_{C}=\vec{W}-\frac{M_{m}-\bar{M}_{m 0}}{\rho} \vec{Y}_{m 0} .
$$

Average statistical weight of particles substituting metal atoms, can be approximately calculated by the following formula:

$$
\bar{M}_{m 0}=\frac{\rho-M_{m} n_{m 0}}{n_{0}-n_{m 0}} .
$$

Here $n_{0}=p_{0} / k T$ is the total concentration of particles in arc column plasma assumed to be isothermal; $p_{0}$ is the atmospheric pressure; $T$ is the plasma temperature; $k$ is the Boltzmann constant. In terms of the above approach, the sum of specific mass flows of atoms of metal and other particles, forming the plasma, is equal to total density of plasma mass flow. This is indicative of consistent description of gas-dynamic and diffusion processes.

Diffusion rate $\vec{w}_{D 0}$ of metal atoms is connected with their concentration $n_{m 0}$ and diffusion flow density $\vec{Y}_{m 0}$ as follows:

$$
\vec{w}_{D 0}=\frac{Y_{m 0}}{n_{n 0}},
$$

where $\vec{Y}_{m 0}$ value in the simplest case can be determined from the following relationship [1]:

$$
\vec{Y}_{m 0}=-\frac{D_{0}}{T} \operatorname{grad}\left(n_{m 0} T\right),
$$

where $D_{0}$ is the coefficient of metal atom diffusion in plasma. 
Substituting (4), (6), (7) into equation (1), we obtain

$$
\frac{d n_{m 0}}{d t}=\operatorname{div}\left[G_{0} \operatorname{grad}\left(n_{m 0} T\right)\right]-n_{m 0} \operatorname{div} \vec{W}-\dot{n}_{m 0},
$$

where $d n_{m 0} / d t$ is the substantial derivative;

$$
G_{0}=\frac{D_{0}}{T}\left(1-n_{m 0} \frac{M_{m}-\bar{M}_{m 0}}{\rho}\right) .
$$

We will perform similar transformations with equations (2), (3) and will additionally allow for drift of charged metal particles (single- and double-charged ions) in the electric field. As a result we will have

$$
\begin{gathered}
\frac{d n_{m 1}}{d t}=\operatorname{div}\left[G_{1} \operatorname{grad}\left(n_{m 1} T\right)+\right. \\
\left.+b_{1} m_{m 1} \operatorname{grad} \varphi\right]-n_{m 1} \operatorname{div} \vec{W}-\dot{n}_{m 1}+\dot{n}_{m 0}, \\
\frac{d n_{m 2}}{d t}=\operatorname{div}\left[G_{2} \operatorname{grad}\left(n_{m 2} T\right)+\right. \\
\left.+b_{2} m_{m 2} \operatorname{grad} \varphi\right]-n_{m 2} \operatorname{div} \vec{W}+\dot{n}_{m 1} .
\end{gathered}
$$

Here, $G_{Z}=\frac{D_{Z}}{T}\left(1-n_{m Z} \frac{M_{m}-\bar{M}_{m Z}}{\rho}\right) ; D_{Z}$ are the coefficients of diffusion of metal ions with charge number $Z(Z=1,2)$;

$$
\bar{M}_{m Z}=\frac{\rho-M_{m} n_{m Z}}{n_{0}-n_{m Z}}
$$

$b_{Z}=\frac{e Z}{k} G_{Z}$ are the mobilities of metal ions in the electric field; $e$ is the electron charge; $\varphi$ is the scalar potential of electric field in the arc column.

Summing up equations (8)-(10), we obtain the equation of metal particle transfer in arc plasma

$$
\begin{gathered}
\frac{d n_{m}}{d t}=\operatorname{div}\left[G_{0} \operatorname{grad}\left(n_{m} T\right)+\right. \\
+\left(G_{1}-G_{0}\right) \operatorname{grad}\left(n_{m 1} T\right)+ \\
+\left(G_{2}-G_{0}\right) \operatorname{grad}\left(n_{m 2} T\right)+ \\
\left.+\left(b_{1} n_{m 1}+b_{2} n_{m 2}\right) \operatorname{grad} \varphi\right]-n_{m} \operatorname{div} \vec{W},
\end{gathered}
$$

where $n_{m}=n_{m 0}+n_{m 1}+n_{m 2}$ is the total concentration of heavy particles of metal vapour.

Let us express the concentrations of metal ions $n_{m Z}(Z=1,2)$ through total concentration of metal particles in the plasma: $n_{m Z}=K_{Z} n_{m}$, where coefficients $K_{Z}$ correspond to first $(Z=1)$ and second $(Z=2)$ ionization of metal atoms. Considering the assumption of local thermodynamic equilibrium of arc column plasma, coefficients
$K_{Z}$ can be determined for equilibrium plasma of specified composition and temperature.

Introducing $\bar{G}_{1}=G_{1}-G_{0} ; \bar{G}_{2}=G_{2}-G_{0}$ designations, we will rewrite equation (11) for total concentration of metal particles in the plasma:

$$
\begin{aligned}
& \frac{d n_{m}}{d t}+n_{m} \operatorname{div} \vec{W}=\operatorname{div}\left[G_{0} \operatorname{grad}\left(n_{m} T\right)+\right. \\
& \left.+\bar{G}_{1} \operatorname{grad}\left(K_{1} n_{m} T\right)+\bar{G}_{2} \operatorname{grad}\left(K_{2} n_{m} T\right)\right]+ \\
& \left.+\operatorname{div}\left[b_{1} K_{1}+b_{2} K_{2}\right) n_{m} \operatorname{grad} \varphi\right] .
\end{aligned}
$$

Equation (12) describes the following kinds of transfer of heavy particles of metal vapour in arc plasma: convective transfer, concentration diffusion, thermodiffusion, as well as vapour ion drift in the electric field. Knowing the solution of this equation, $n_{m}$ and considering the taken assumption of local thermodynamic equilibrium of arc column plasma, its ionization composition can be determined through application of the respective system of Saha's equations, law of partial pressures and condition of plasma quasineutrality [14]. The thus calculated concentrations of particles of multicomponent arc column plasma can be used for calculation of its thermodynamic and transport properties [18], included into complex model equations [14].

We will define boundary conditions for diffusion equation (12). Assuming that the arc column is characterized by axial symmetry, we will introduce the cylindrical system of coordinates $(r, z)$ and consider design region $\Omega=\{0<r<$ $<R, 0<z<L\}$, shown in Figure 1.

Considering the direction of movement of shielding gas and plasma in near-cathode zone of arc column [17], we will assume that particles of evaporated anode metal do not reach plane

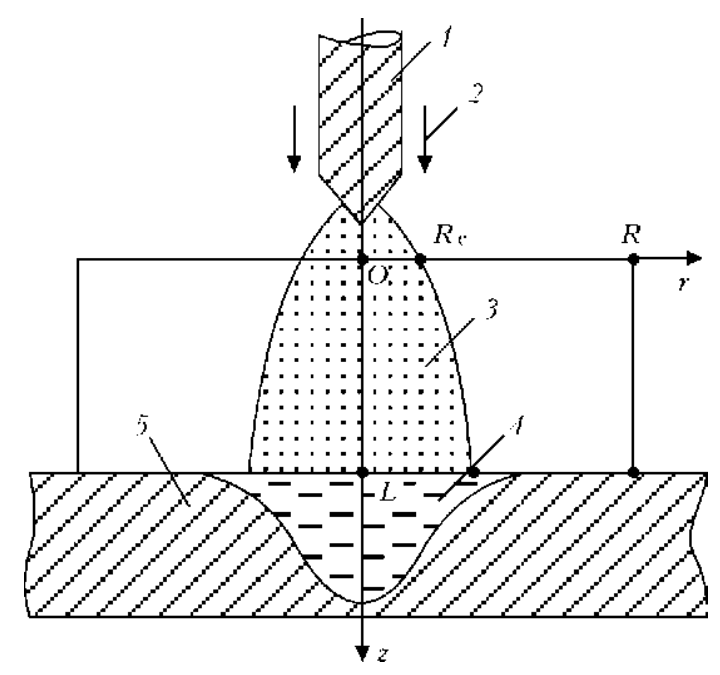

Figure 1. Schematic for mathematical description of arc plasma: 1 - refractory cathode; 2 - shielding gas, 3 arc column plasma; 4 - molten (evaporating) metal; 5 anode 
$z=0$ (see Figure 1), i.e. on upper limit of design region we will set

$$
\left.n_{m}\right|_{z=0}=0 .
$$

On the arc axis (at $r=0$ ) we will assume the following conditions of symmetry:

$$
\left.\frac{\partial n_{m}}{\partial r}\right|_{r=0}=0 .
$$

On the outer limit of design region (at $r=R$ ) we will assign «soft» boundary conditions

$$
\begin{gathered}
\left.n_{m}\right|_{r=R}=0 \text { at } W_{r}(R, z) \leq 0 ; \\
\left.\frac{d n_{m}}{d t}\right|_{r=R}=0 \text { at } W_{r}(R, z)>0,
\end{gathered}
$$

where $W_{r}(r, z)$ is the radial component of the vector of average mass velocity of plasma.

On the boundary of multicomponent plasma with anode layer (at $z=L$ ) we can write the boundary condition in the following form:

$$
n_{m} \mid z=L=n_{m 0}^{L}(r)+n_{m 1}^{L}(r)+n_{m 2}^{L}(r),
$$

where $n_{m Z}^{L}(r)=n_{m Z}(r, L)$ are the respective distributions of concentration of metal vapour particles, being in charge state $Z$, which can be determined according to model of anode region of the arc with evaporating anode [14], depending on local values of near-anode plasma temperature and anode surface temperature, its evaporation mode, as well as kind of shielding gas. Limiting further consideration to diffusion evaporation mode, it can be assumed with sufficient accuracy that local values of partial pressure of atoms and ions of metal component of plasma $p_{m}$ on the above boundary are equal to pressure of saturated vapour of anode metal at the respective value of temperature on its surface $T_{\mathrm{a}}$ :

$$
\left.p_{m}\right|_{z=L}=p_{0} \exp \left\{\frac{\lambda_{v}}{k}\left[\frac{1}{T_{B}}-\frac{1}{T_{\mathrm{a}}(r)}\right]\right\},
$$

where $\lambda_{v}$ is the energy consumed for transition of one metal particle from the liquid into the vapour phase; $T_{B}$ is the boiling temperature of anode metal.

Model of convective diffusion of ionized metal vapour (12)-(17) is a component part of complex model of processes of energy, pulse, mass and charge transfer in multicomponent plasma of the column and anode region of electric arc in inertgas nonconsumable-electrode welding [14]. For numerical simulation of heat, gas-dynamic and electromagnetic processes in such plasma we will use equations of single-temperature model [17]. When allowing for evaporation of anode metal on the boundary of condensed phase with arc plasma, there exists a diffusion flow of metal vapour, as a result of which the axial component of plasma velocity vector on this boundary is not equal to zero (unlike the condition of «sticking», used in [17] for the case of water-cooled anode). Considering the fact that atoms and ions of shielding gas, which is inert, cannot accumulate on the anode surface, the resulting flow of heavy particles of gas near the anode surface can be considered to be equal to zero. Then, allowing for diffusion and convective mechanisms of metal vapour particle transfer, the boundary condition for axial component of vector of average mass velocity of plasma on the boundary of arc column with the anode region (at $z=L$ ) can be written in the following form:

$$
\left.W_{z}\right|_{z=L}=\frac{M_{m}\left[Y_{m 0_{z}}^{L}(r)+Y_{m 1_{z}}^{L}(r)+Y_{m 2_{z}}^{L}(r)\right]}{\rho(r, L)-M_{m}\left[n_{m 0}^{L}(r)+n_{m 1}^{L}(r)+n_{m 2}^{L}(r)\right]} .
$$

Here $Y_{m Z_{Z}}^{L}(r)$ are the respective distributions of axial components of densities of diffusion flows of metal atoms and ions, which are in charged state $Z$.

Simulation results and their discussion. For numerical analysis of the influence of diffusion evaporation of anode metal on processes of energy, mass and charge transfer in anode region and column of the considered arc we will assign anode surface temperature distribution by normal-circular law $T_{\mathrm{a}}(r)=\left(T_{\mathrm{a} 0}-T_{\infty}\right) \exp \left(-a^{2} r^{2}\right)+$ $+T_{\infty}$, where $T_{\mathrm{a} 0}$ is the temperature on the axis of the region of arc anode binding; $T_{\infty}$ is the temperature of metal surface at a distance from the above region. We will select coefficient of concentration $a$ so that diameter of molten zone on anode surface was $5 \mathrm{~mm}$. Characteristic profiles of $T_{\mathrm{a}}(r)$ distribution at $T_{\infty}=500 \mathrm{~K}$ are shown in Figure 2.

Numerical simulation of characteristics of multicomponent plasma of the column and anode region of electric arc with tungsten cathode and evaporating anode from low-carbon steel was conducted at the following parameters: arc length $L=2.9 \mathrm{~mm}$; arc current $I=200 \mathrm{~A}$; shielding gas is argon, and evaporating element is iron. Initial distributions of arc column plasma characteristics, required to solve the non-stationary problem [17], together with equation (12), were assigned as specified in [17]; initial concentration of metal vapour in the arc gap was also taken to be zero. Calculations were performed right up to establishment of steady state of arc plasma.

We will introduce $\gamma=n_{m} /\left(n_{q}+n_{m}\right)$ designation, where $n_{g}=n_{g 0}+n_{g 1}$ is the total concentra- 
tion of heavy particles (atoms and ions) of shielding gas, and will consider the distribution of the fraction of heavy metal particles $\gamma$ in arc column plasma for two variants of distribution of evaporating anode surface temperature, which correspond to $T_{\mathrm{a} 0}=2600 \mathrm{~K}$ (Figure $3, a$ ) and $T_{\mathrm{a} 0}=$ $=3065 \mathrm{~K}$ (Figure 3, b). At $2500-2600 \mathrm{~K}$ temperature of molten anode metal, evaporated metal particles appear above its surface, their content reaching $10 \%$ (see Figure $3, a$ ). At increase of temperature in the center of the region of arc anode binding above $3000 \mathrm{~K}$, mass flow of vapour from anode surface into arc column increases, that results in formation of a region of arc plasma with high (up to $80 \%$ ) content of metal vapour (Figure 3,b).

Field of concentration of evaporated metal particles $n_{m}$ in near-anode plasma forms as a result of interaction of the following four factors: diffusion and convective transfer of metal particles from anode surface into arc column; arc plasma flow with low content of metal vapour, moving over the anode; transfer of metal vapour particles towards the anode due to thermodiffusion; drift of charged metal particles (single- and double-charged ions) in the electric field. Distribution of the fraction of heavy iron particles in the considered arc plasma, presented in Figure 3 , is the result of competing interaction of the above-mentioned four transfer mechanisms. Two characteristic features of evaporated metal particle distribution in near-anode plasma can be singled out. On the one hand, convective flow of plasma from near-cathode region of the column, containing practically no metal vapour, is trying to oust metal vapours from evaporation zone in the radial direction. As a result, width of near-surface plasma layer, containing a noticeable quantity of metal vapour $(\gamma>3 \%)$, turns out to be 1.5-2 times greater than the radius of molten zone on anode surface, and thickness of

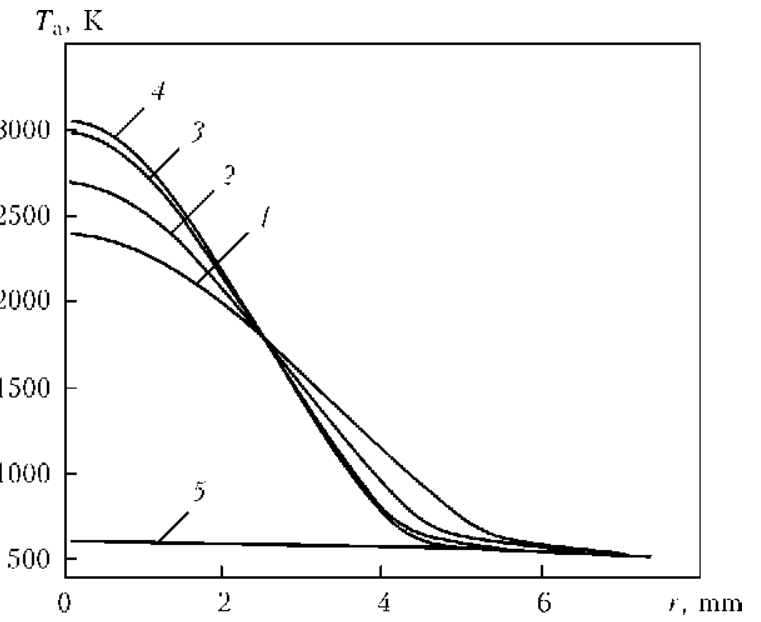

Figure 2. Temperature distribution of anode surface in the region of arc anode binding (here and in Figures 4-7: 1 $T_{\mathrm{a} 0}=2400 \mathrm{~K} ; 2-2700 ; 3-3000 ; 4-3065 ; 5$ - watercooled (nonevaporating) anode)

this layer is $0.3-0.5 \mathrm{~mm}$. As thickness of the region taken up by vapour is small compared to arc length, influence of evaporated metal in the considered case is limited to just near-anode region of the arc and practically does not affect the processes of heat, mass and charge transfer in it is column. At the same time, region of nearanode plasma, the most enriched in iron vapours, turns out to be «cut off» from the anode surface. This effect can be explained as follows. Ionization composition of metal vapour, which comes to near-anode region of arc column and is further on transported into the region with higher plasma temperature, undergoes changes due to intensive ionization of metal atoms. On the other hand, because of low diffusion mobility of metal ions, they accumulate in the above region, that is exactly what causes formation of a zone of maximum content of metal vapour, localized at a certain distance from anode surface.

Diffusion evaporation of anode metal has the greatest influence on such characteristics of anode region of the considered arc as fraction of iron

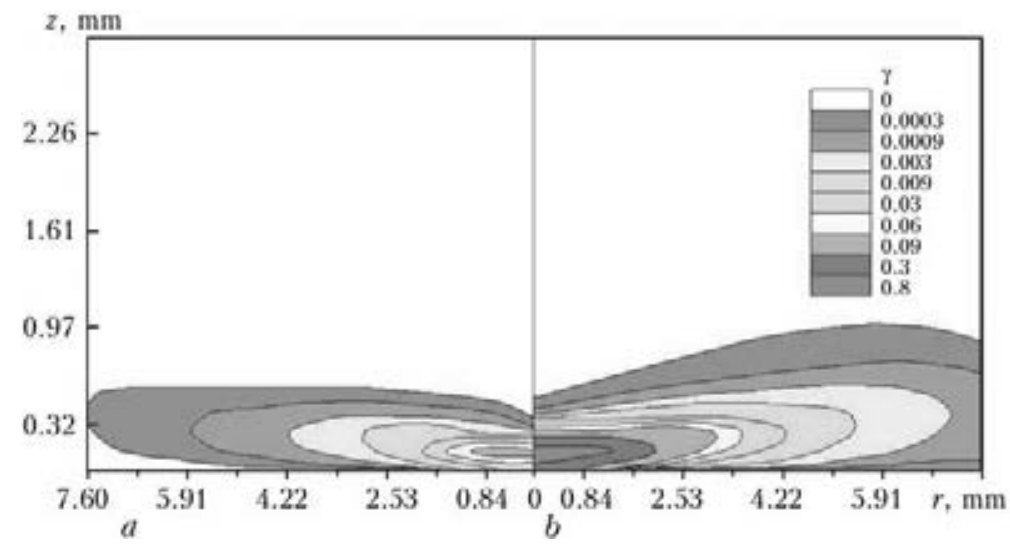

Figure 3. Distribution of fraction of heavy iron particles in near-anode region of arc plasma column: $a-T_{\mathrm{a} 0}=2600 \mathrm{~K}$; $b-3065$ 


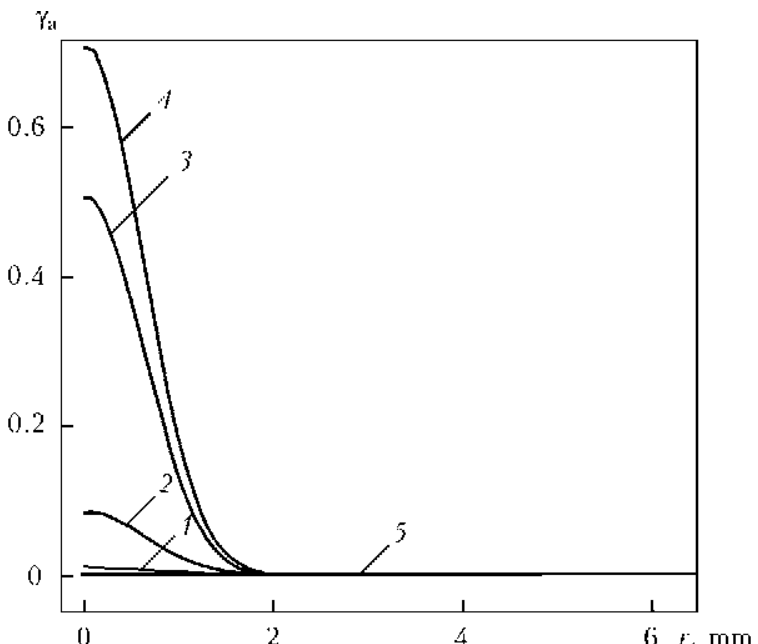

Figure 4. Radial distribution of fraction of heavy iron particles in multicomponent near-anode plasma

particles in near-anode plasma $\gamma_{\mathrm{a}}(r)=\gamma(r, L)$ and its temperature $T_{p \mathrm{a}}(r)=T(r, L)$, electric current density $j_{\mathrm{a}}(r)$ and heat flow density $q_{\mathrm{a}}(r)$ on anode surface. Let us consider the influence of temperature on evaporating anode surface on distribution of the above characteristics in the region of anode binding of the arc. Figures 4-7 give the results of calculations of $\gamma_{\mathrm{a}}, T_{p \mathrm{a}}, j_{\mathrm{a}}$ and $q_{\mathrm{a}}$ for different thermal states of anode surface.

Maximum content of metal vapour is reached on the axis of near-anode plasma layer, increasing with temperature rise on anode surface in the center of the region of arc anode binding (see Figure 4). Here, maximum value of average mass velocity of vapour motion $\left|W_{z}(0, L)\right|$ near anode surface also rises at increase of the above temperature. So, for instance, at $T_{\mathrm{a} 0}=3065 \mathrm{~K}$ this velocity can reach more than $10 \mathrm{~m} / \mathrm{s}$. Such intensive flow of relatively cold vapour, moving from anode surface into arc column, causes local freezing of near-anode plasma. This effect is manifested in that part of anode region, which is lo-

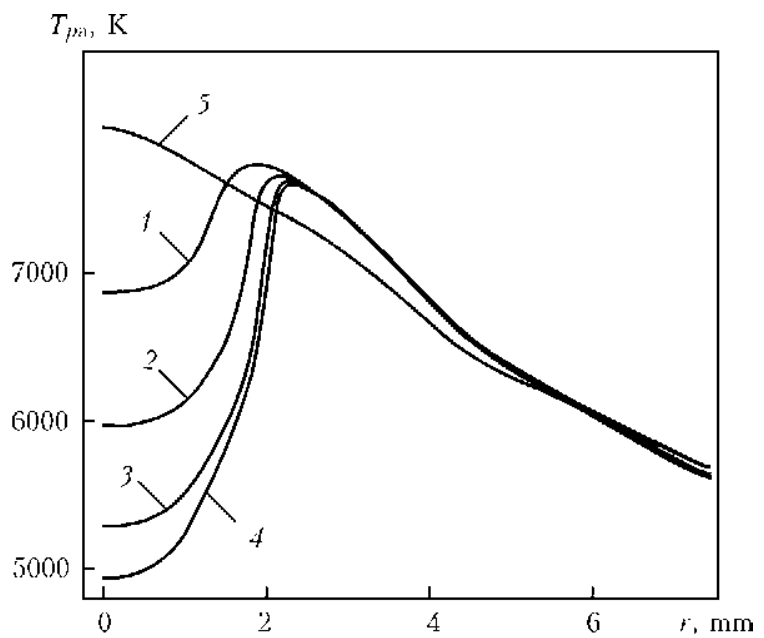

Figure 5. Radial distribution of arc column plasma temperature on the interface with anode region

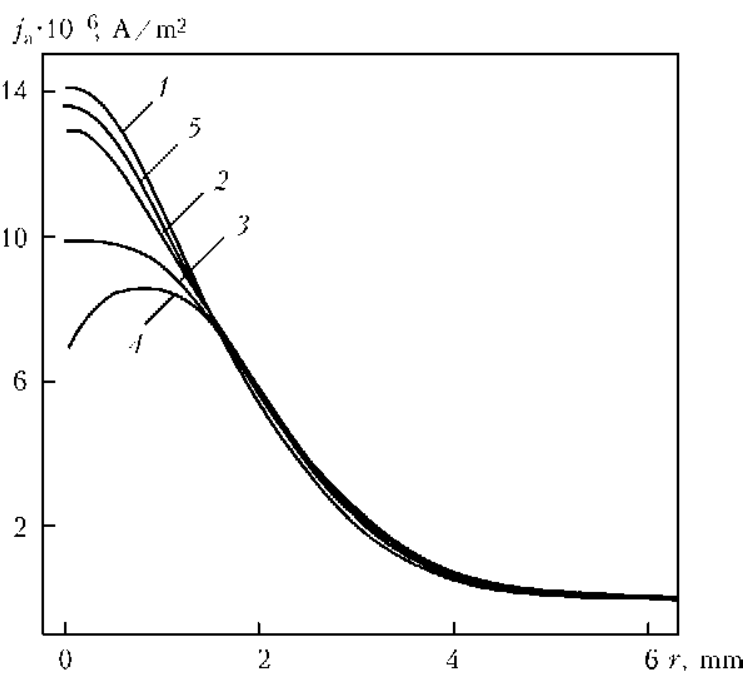

Figure 6. Radial distributions of electric current density on anode surface

cated above the most heated zone of molten anode metal surface, and the more so, the higher the surface temperature in this zone (see Figure 5).

Despite the fact that increase of concentration of easily ionized (compared to argon) metal vapour in multicomponent plasma with $T_{\mathrm{a} 0}$ rise should lead to increase of its electric conductivity $\sigma$, the above-noted effect of local freezing of nearanode plasma by vapour flow has a more significant role, leading to lowering of $\sigma$ and of electric current density in near-axis zone of the region of arc anode binding, respectively (see Figure 6).

Density of heat flow, applied by the arc to evaporating anode, behaves in a similar fashion (see Figure 7). Considerable lowering of $q_{\mathrm{a}}$ value at high values of anode metal surface temperature is related to reduction of convective energy flow from arc column, as a result of respective variation of gas-dynamic and electromagnetic situation in near-anode region of arc plasma, as well as reduction of heat flow transported to the anode

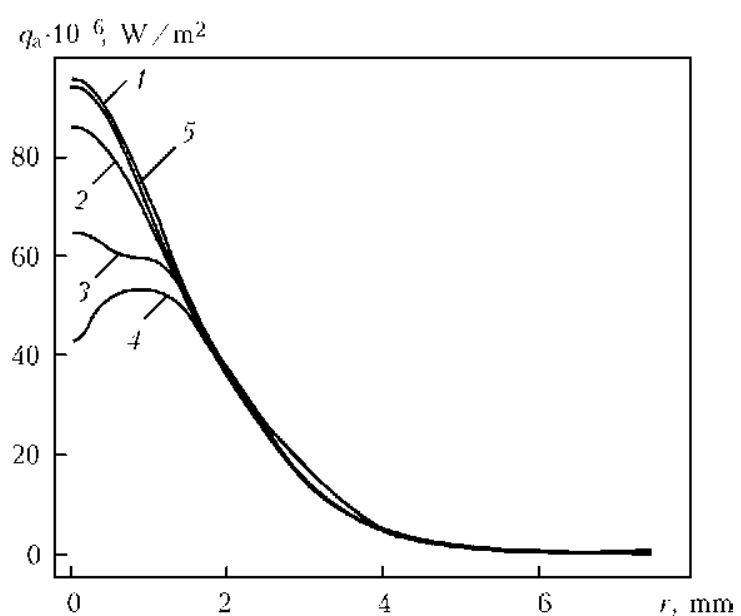

Figure 7. Radial distributions of density of heat flow applied by the arc to the anode 


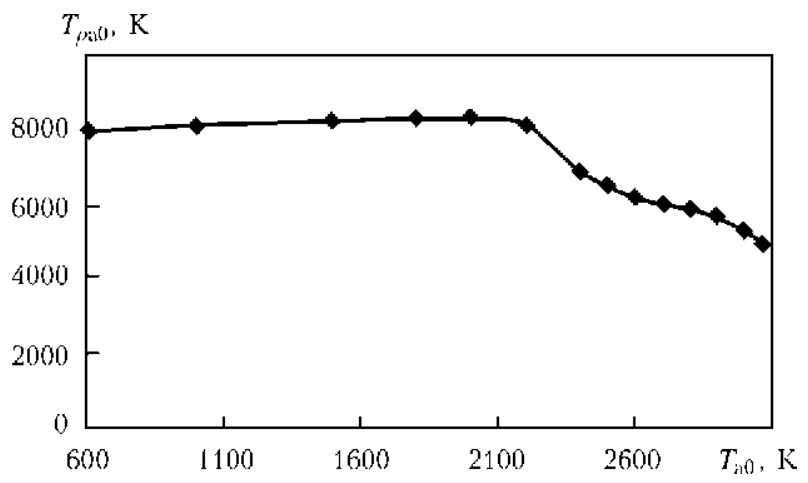

Figure 8. Dependence of axial value of arc plasma temperature on anode region interface on anode surface temperature in the center of the region of arc anode binding

by charged particles, at the expense of respective reduction of $j_{\mathrm{a}}$ (see Figure 6).

Now let us analyze dependencies of axial values of the considered characteristics on anode surface temperature in the center of the region of arc anode binding. Variation of $T_{p \mathrm{a} 0}=T(0$, $L), j_{\mathrm{a} 0}=j_{\mathrm{a}}(0, L)$ and $q_{\mathrm{a} 0}=q_{\mathrm{a}}(0, L)$ with $T_{\mathrm{a} 0}$ rise is shown in Figures 8-10. Range of variation of maximum surface temperature of molten anode metal studied in this work can be conditionally divided into two intervals: $T_{\mathrm{a} 0}<2400 \mathrm{~K}-$ corresponds to nonevaporating anode; $2400 \mathrm{~K}<$ $<T_{\mathrm{a} 0}<3100 \mathrm{~K}-$ corresponds to diffusion mode of anode metal evaporation. In the first temperature range all the characteristics of arc anode region weakly depend on $T_{\mathrm{a} 0}$, whereas in the second range an essential lowering of $T_{p \mathrm{a} 0}$ takes place. As regards $j_{\mathrm{a} 0}$ and $q_{\mathrm{a} 0}$, they behave nonmonotonically (see Figures 9 and 10). Initially observed reduction of electric current density and density of heat flow entering the anode on the axis of the region of arc anode binding is replaced by their certain increase, so that at $T_{\mathrm{a} 0} \approx 2800 \mathrm{~K}$ these values reach their local maximums. Their further lowering proceeds the faster, the more intensive is anode metal evaporation. The noted peculiarity is the most pronounced in the behaviour of such an integral characteristic of thermal interaction of arc plasma with anode metal as

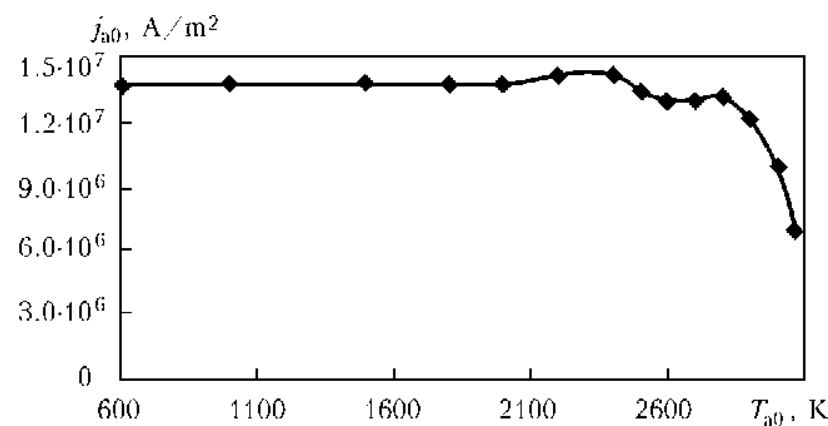

Figure 9. Dependence of axial value of density of electric current on the anode on its surface temperature in the center of the region of arc anode binding

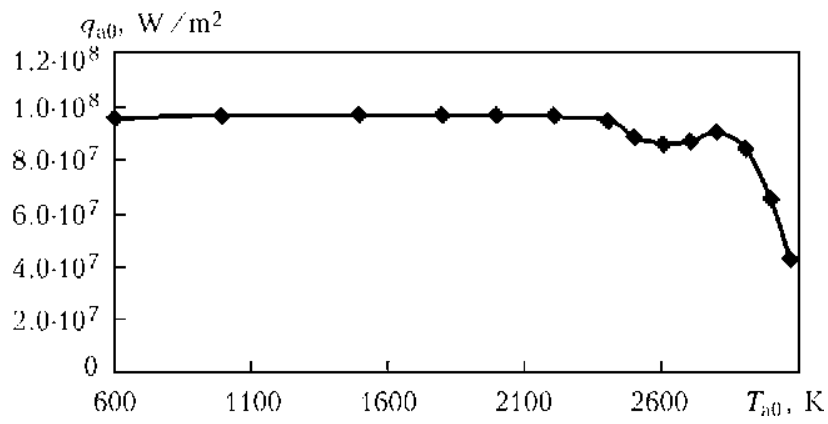

Figure 10. Dependence of axial value of density of heat flow to the anode on its surface temperature in the center of the region of arc anode binding

complete heat power $P$ applied by the arc ( $\mathrm{Fi}^{-}$ gure 11).

Dependence of density of heat losses of anode metal for evaporation in near-axis zone of the region of the arc anode binding $q_{v 0}$ on its surface temperature in this zone is shown in Figure 12. As follows from calculation data given in this Figure, at $T_{\mathrm{a} 0}$ rise up to $3000 \mathrm{~K}$, the above value can be equal to about $25 \%$ of the respective value of heat flow applied by arc plasma to evaporating anode (compare Figures 10 and 12), and it should be taken into account at determination of energy balance of its surface.

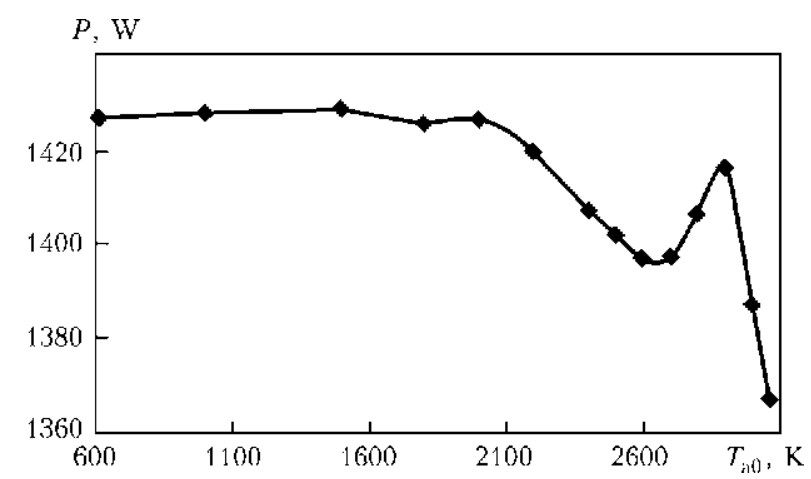

Figure 11. Dependence of heat power applied by the arc to the anode on anode surface temperature in the center of the region of arc anode binding

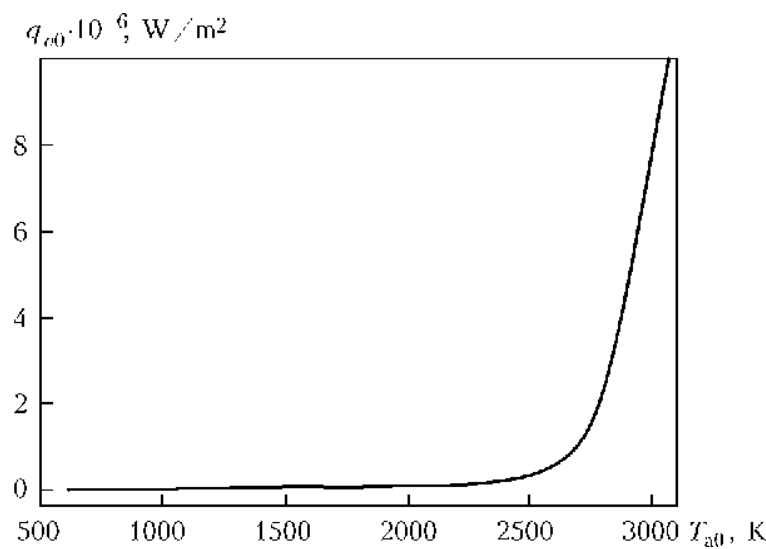

Figure 12. Dependence of axial value of density of energy losses for anode metal evaporation on its surface temperature in the center of the region of arc anode binding 
On the whole, numerical analysis of the influence of diffusion evaporation of anode metal on characteristics of the column and anode region of the arc with refractory cathode, running in inert gas, which was conducted in this work, leads to the following conclusions:

1. In the case of inert-gas nonconsumable-electrode arc welding the influence of evaporated anode material on characteristics of arc column plasma is manifested only in thin (just up to $0.5 \mathrm{~mm}$ ) layer adjacent to anode region. As regards arc plasma characteristics in the rest of arc column, they practically do not change, compared to the arc running to water-cooled (nonevaporating) anode.

2. Evaporation of metal being welded leads to an essential restructuring of spatial distributions of plasma characteristics of anode region of welding arc with nonconsumable electrode, as well as characteristics of its thermal and electromagnetic interaction with weld pool surface. In particular, with increase of melt surface temperature in the center of the region of arc anode binding, density of heat flow applied by the arc to the item being welded and density of electric current on its surface decrease. Alongside losses of molten metal energy for evaporation, this leads to lowering of effectiveness of arc heating of the metal being welded.

1. Murphy, Ant.B. (2010) The effects of metal vapour in arc welding. J. Phys. D: Appl. Phys., 43.

2. Hsu, K.C., Etemadi, K., Pfender, E. (1983) Study of the free-burning high-intensity argon arc. J. Appl. Phys., 54(3), 1293-1301.

3. Hsu, K.C., Pfender, E. (1983) Two-temperature modeling of the free-burning high-intensity arc. Ibid., 54(8), 4359-4366.

4. Engelsht, V.S., Gurovich, V.Ts., Desyatkov, G.A. et al. (1990) Low-temperature plasma. Vol. 1: Theory of electric arc column. Novosibirsk: Nauka.
5. Zhu, P., Lowke, J.J., Morrow, R. et al. (1995) Prediction of anode temperatures of free burning arcs. $J$. Phys. D: Appl. Phys., 28, 1369-1376.

6. Jenista, J., Heberlein, J.V.R., Pfender, E. (1997) Numerical model of the anode region of high-current electric arcs. IEEE Transact. on Plasma Science, 25(5), 883-890.

7. Lowke, J.J., Morrow, R., Haidar, J. (1997) A simplified unified theory of arcs and their electrodes. $J$. Phys. D: Appl. Phys., 30, 2033-2042.

8. Haidar, J. (1999) Non-equilibrium modeling of transferred arcs. Ibid., 32, 263-272.

9. Sansonnets, L., Haidar, J., Lowke, J.J. (2000) Prediction of properties of free burning arcs including effects of ambipolar diffusion. Ibid., 33, 148-157.

10. Nishiyama, H., Sawada, T., Takana, H. et al. (2006) Computational simulation of arc melting process with complex interactions. ISIJ Int., 46(5), 705-711.

11. Lago, F., Gonzalez, J.J., Freton, P. et al. (2004) A numerical modeling of an electric arc and its interaction with the anode. Pt 1: The two-dimensional model. Ibid., 37, 883-897.

12. Tanaka, M., Yamamoto, K., Tashiro, S. et al. (2008) Metal vapour behaviour in gas tungsten arc thermal plasma during welding. Welding in the World, $52(11 / 12), 82-88$.

13. Mougenot, J., Gonzalez, J.J., Freton, P. et al. (2013) Plasma-weld pool interaction in tungsten inert-gas configuration. J. Phys. D: Appl. Phys., 46, 135-206.

14. Krivtsun, I.V., Demchenko, V.F., Krikent, I.V. (2010) Model of the processes of heat-, mass- and charge transfer in the anode region and column of the welding arc with refractory cathode. The Paton Welding J., 6, 2-9.

15. Almeida, R.M.S., Benilov, M.S, Naidis, G.V. (2000) Simulation of the layer of non-equilibrium ionization in a high-pressure argon plasma with multiply-charged ions. J. Phys. D: Appl. Phys., 33, 960-967.

16. Krivtsun, I.V., Krikent, I.V., Demchenko, V.F. (2013) Modelling of dynamic characteristics of a pulsed arc with refractory cathode. The Paton Welding J., 7, 13-23.

17. Krikent, I.V., Krivtsun, I.V., Demchenko, V.F. (2012) Modelling of processes of heat-, mass- and electric transfer in column and anode region of arc with refractory cathode. Ibid., 3, 2-6.

18. Krivtsun, I.V., Porytsky, P., Demchenko, V. et al. (2010) On the application of the theory of Lorentzian plasma to calculation of transport properties of multicomponent arc plasmas. Europ. Phys. J. $D, \mathbf{5 7}, 77-85$. 